\title{
Robot Systems for Residential Building Construction: Integration and Operation
}

\author{
Prof. Dr.-Ing. D. Spath, Prof. Dr.-Ing. J. Schmidt, Dipl.-Ing. J. Andres \\ Institute for Machine Tools and Production Science (wbk) \\ University of Karlsruhe (TH), Kaiserstrasse 12, D-76128 Karlsruhe, Germany
}

\section{Abstract}

The article deals with the operation of robot systems for the onsite construction of residential buildings and their integration in a semi-automated construction process. On one hand the integration and necessary work preparation tasks will be described for an mobile onsite masonry robot developed in the European project ROCCO (Robot Assembly System for Computer Integrated Construction) as well as the operation of the system itself. On the other hand a description of the operation of a conceptuated robot for interior finishing works will be given. Especially, the programming methods are focussed that the aim of a userfriendly application can be achieved and a medium range realisation is enabled.

\section{Motivation}

Because of an increasing pressure on construction costs especially in Germany it will be necessary in the near future to integrate higher automated systems to meet rationalisation and humanisation as well. Although, the demand on residential buildings is still on a high level private investment is inhibited by a strong rise of construction costs compared to the average costs of living standard [1,2].

Besides the actual mechanical development of kinematics, actuators and effectors of the robot systems for masonry construction and single interior construction tasks an integrated work preparation for the robot programming and user-friendly operator control systems must be created to aim an efficient construction process. Therefore, as far as the processes are concerned it has to be considered if the design of integrated CAD/CAM processes are feasible or if the preconditions for highly integrated data processings are not given. The most important reason is the mainly small and medium sized structure of the building industry for these 
different construction tasks. So, one of the most essential parts for the operation is the generation of the robot programmes shown examplarily for the masonry robot ROCCO and the interior finishing robot especially for preparation of electrical installation tasks.

\section{$2 \quad$ Realised and Conceptuated Robot Systems}

\subsection{The Masonry Robot System ROCCO}

The main structure of the realised masonry robot system consists of an omnidirectional, mobile platform for any desired movements on floors, the hydraulic manipulator for the wall assembly in respective working positions and a fault tolerant assembly tool for gripping and positioning of blocks. The kinematical structure consists of three main rotational axes and two hand axes. The resulting working room in the wall amounts to $\pm 3.6 \mathrm{~m}$ in width and $4.5 \mathrm{~m}$ in height. The manipulator is designed for a handling weight of $400 \mathrm{~kg}$ so it is possible to handle all occuring bricks, concrete and sandlime blocks. The hydraulically driven mobile platform is supplied with the laser navigation system CAPSY for the measurement of the different working positions $[3,4,5]$.

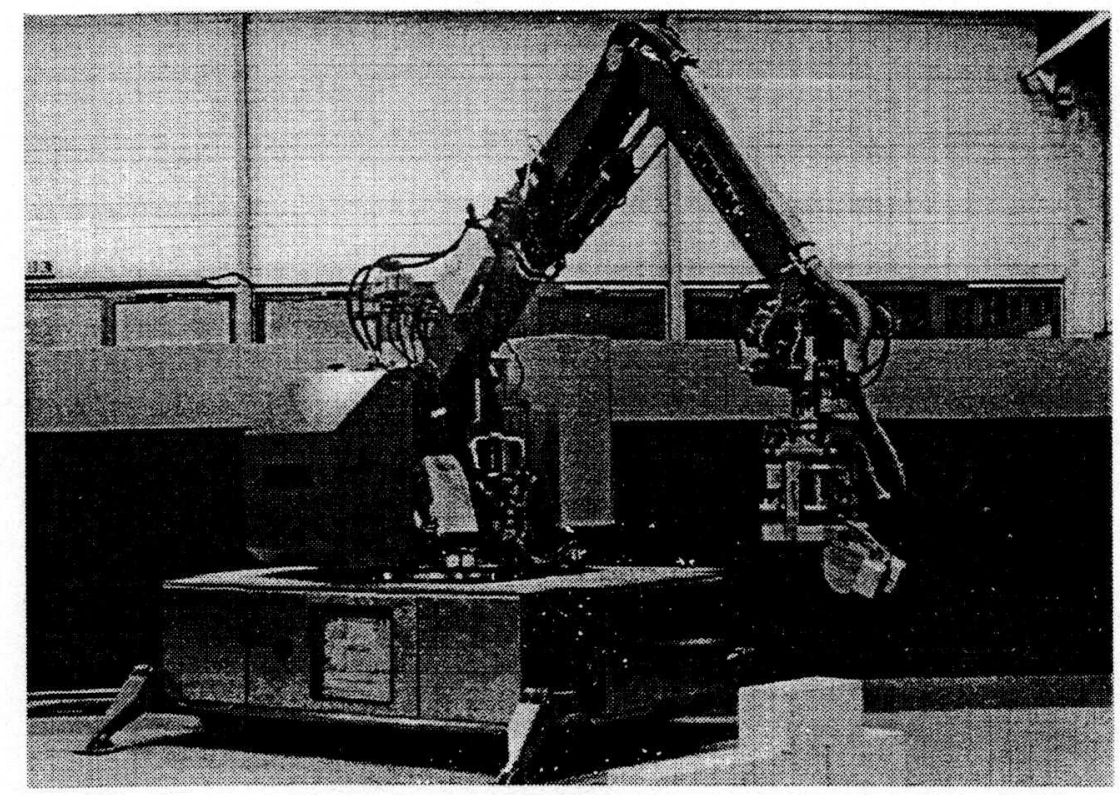

Fig. 1: masonry robot $R O C C O$ 


\subsection{The Interior Finishing Robot}

Due to the tasks to operate at walls to complete the interior of buildings the kinematical structure consists of three main linear axes. Therefore, to pass doors and to adapt to different ceiling heights the vertical axis is devided into two parts. To realise also different working widthes the horizontal axis can be exchanged. Further kinematical demands result from the necessity to reach corners of rooms very closely. Because of existing clearance volumes by linear axis a special developed wrist to bridge these volumes is designed. The mechanical structure of the wrist consists of three actuators implemented with hollow shaft drives that are tilted $45^{\circ}$ against the horizontal. The possible working areas at walls amounts to $3 \mathrm{~m}$ in height and $2 \mathrm{~m}$ in width [6].

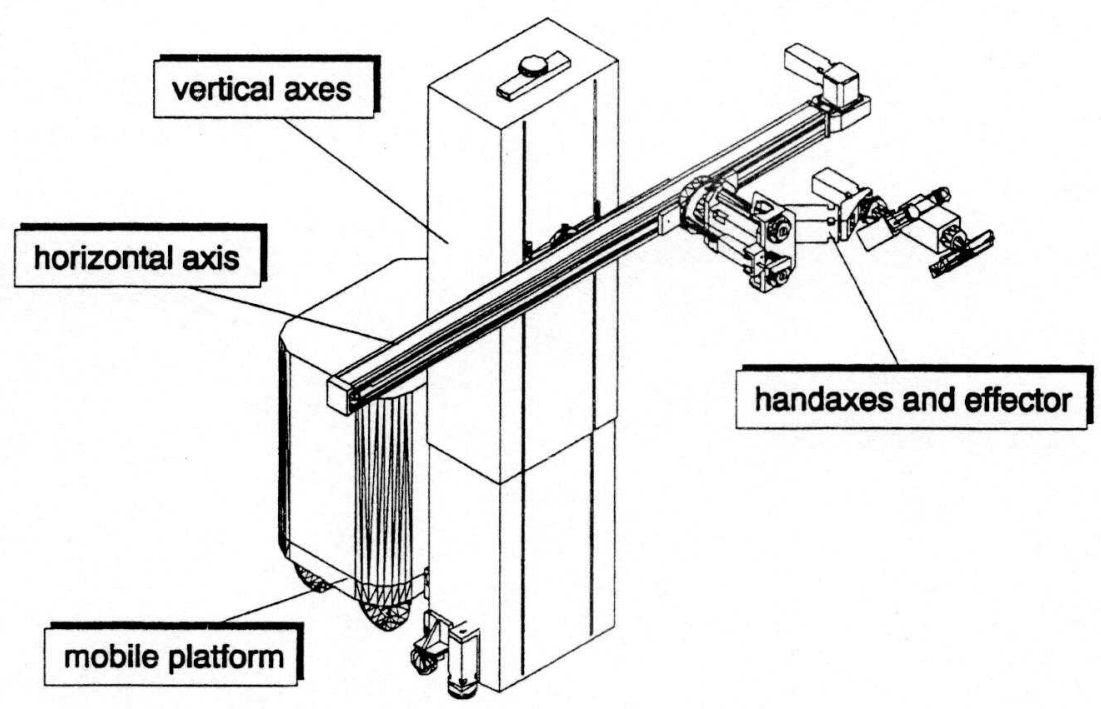

Fig. 2: structure of the indoor robot

\section{Integration of the Robots in the Construction Process}

The focus of integration of the described robot systems is the reduction of the over-all time of construction. In the opposite of the common construction process in practice it is necessary to get more parallel processes on the construction site. Furthermore, stop periods of the robots have to be minimized to aim their economic efficiency. In order to get more processes in parallel a well organized logistics is essential. 
The origin for the work preparation of the construction tasks is formed by the building design of the architect that can be used in different ways. The way to process the generated data will depend on the circumstance if an computer integrated data flow is possible according to different prevailing conditions in the building industry. Additionally, it has to be also faced to increase the efficiency of the robot systems by the use of checklists towards an automationfriendly construction. In particular, a rastered dimensioning of masonry has to be applied for a simpler block handling and a reduced effort in prefabrication.

In the case of the masonry construction as main part of the bare brick work a computer integrated data management provides the most appropriate solution. Here, the precondition of an computer integrated construction process is given because the use of CAD-systems is customized in the early design phase. The main parts of work preparation are the division of the building into walls and single blocks, the layout planning of the construction site and the determination of the need of blocks in the respective working positions. As a result of these planning activities the data for the block pre-fabrication and palletising can be generated [7]. Consequently, all conditions for an automated generation of the robot programmes are complied. After all, the logistics for material supply and the installation of the robot can be initiated for the onsite construction process.

Based on the work process of the masonry construction and the completion of each floor the beginning of post processing tasks like electrical installations can be determined for a parallel execution. In the case of these post processing construction tasks a planning on the construction site seems to be best applicable. The most important reason is that the employment of robot systems for interior finishing (e.g. installations, roughcasting, painting, etc.) actually cannot be based on a totally computer integrated work planning process because of the mostly small sized structure of the building industry in this area. Moreover, if a direct CAD/CAM connection with the produnct design process is not necessary for the system's employment it can also be used as a single solution. Therefore, an onsite work preparation with a high support by a computer aided robot programming system is feasible. So, the qualification of the skilled worker will be used optimally for the onsite planning tasks and as a result of the engineering data the generation of the necessary robot's actions will be supported by the programming system in a highly automated manner. 


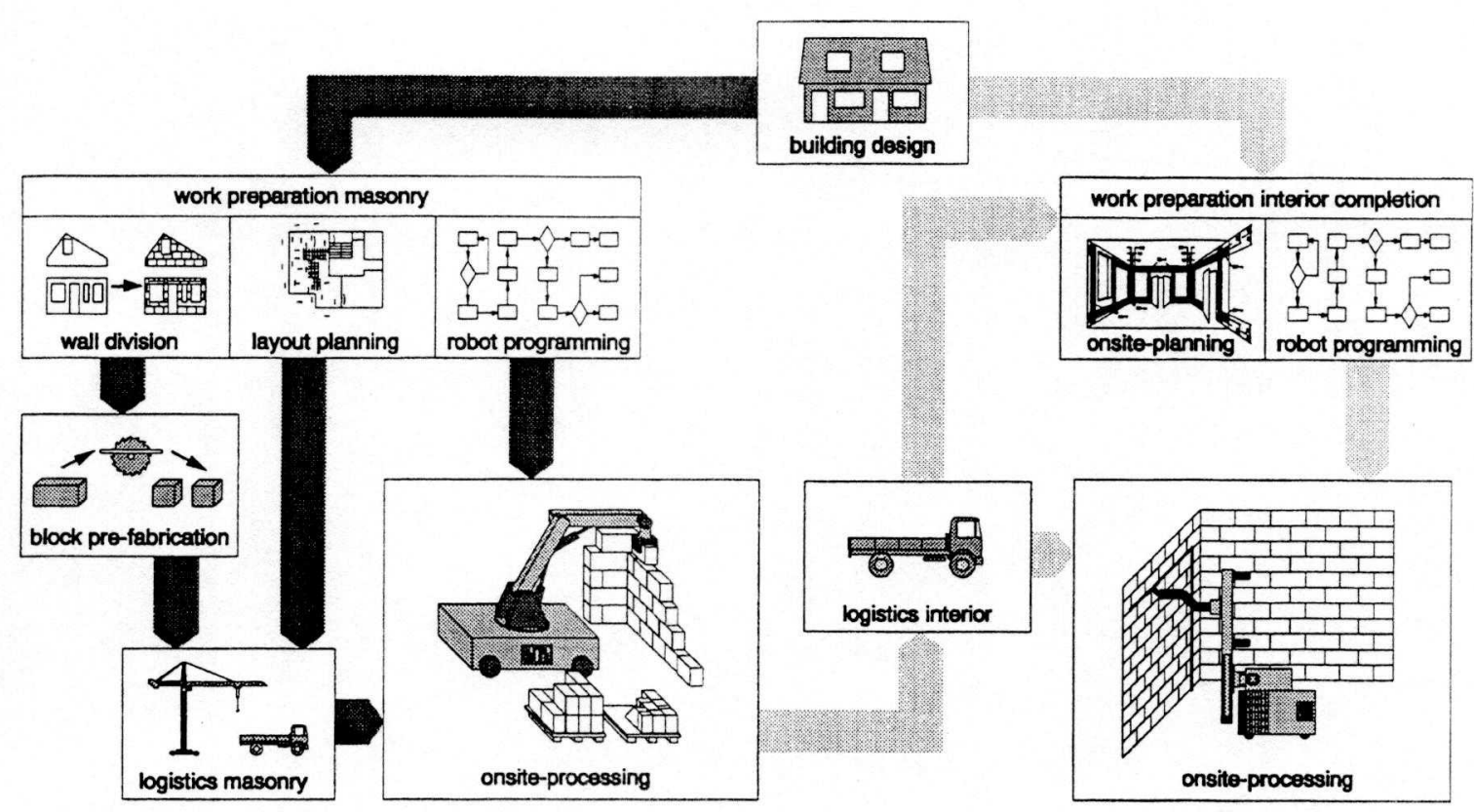

Fig. 3: integration of the robot systems in the construction process

\section{Development of Respective Programming Methods}

\subsection{Communication on the Construction Site}

For an optimized, automated path planning and generation of the respective robot programmes for the masonry as well as for the wall processing application it is unalterable to devide the operation sequences in different functional macros that are adapted to the different occuring cases of assembly and manufacturing procedures. On the basis of this structured organization of the robot operations a controlled execution of sequences and feedback are enabled. Moreover, this kind of direct communication between the PC as operator station and the robot control on the construction site leads to an easier intervention of the operator in the automated process and consequently to an easier handling of occuring errors.

The construction site database as basic element includes all geometric data as well as the data of the layout planning. So, all starting positions and end positions of e.g. blocks or wall slits are stored for further processing. The difference between the masonry application and the wall processing application lays in the generation of the data. A computer integrated work preparation system is underlained for masonry construction whereas the data for e.g electrical installation tasks are generated by the electrician or construction worker on the site. 


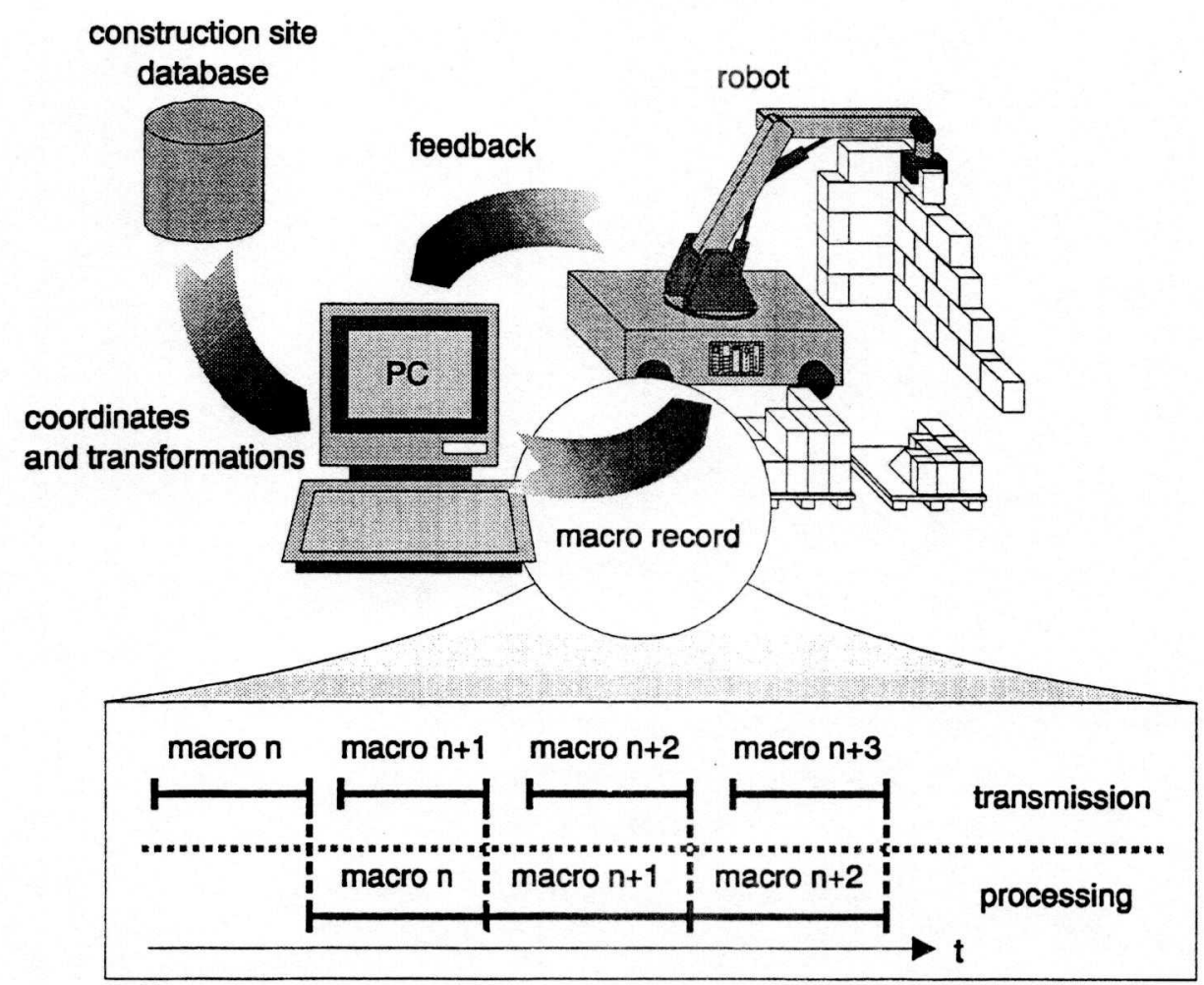

Fig. 4: interaction of the automated construction process

On the side of the robot control all unparameterized functional macros including technological data of the effectors are stored depending on different cases of assembly or manufacturing procedures. On the part of the PC an automated path planning for the manipulator's actions is carried out. Therefore rulebased algorithms undertake the determination of the assembling and manufacturing strategies and their resulting cases, the tranformations between existing coordinate systems and the depending determination of all coordinates for indermediate path points, for course and fine positioning.

In order to avoid attendance times of the robot system the calculation and transmission of parameters are done in advance during the previous macro is processed by the robot. Therefore, the macros are also structured in a way that the time for transmission and feedback is always shorter than the time for the processing itself. 


\subsection{Masonry Programming System}

For the programming of the masonry robot an PC-based, automated offline programming system has been developed at the institute for the path planning of the manipulator part of the robot and the positioning of blocks. Moreover, different strategies depending on different cases of block positions in the wall have to be taken into account. Furthermore, pallet positions, block on pallet locations and the category of blocks have to be considered for the implementation of rule based strategies of robot actions. Because of those different dependencies a useful splitting of a single operating sequence into the following functional macros is done. Firstly, the robot retracts to the minimum radius within the platform dimensions, turns on this radius and execute a linear, coarse positioning towards the pallet, the wall or the mortar device as well. Secondly the fine positioning will be done through relative movements depending on the type of block (in particular regular or irregular) and the type of position (in particular straight or corner). Further two operating tasks are the grasping and the lifting up of blocks from the pallet and their releasing at the wall.

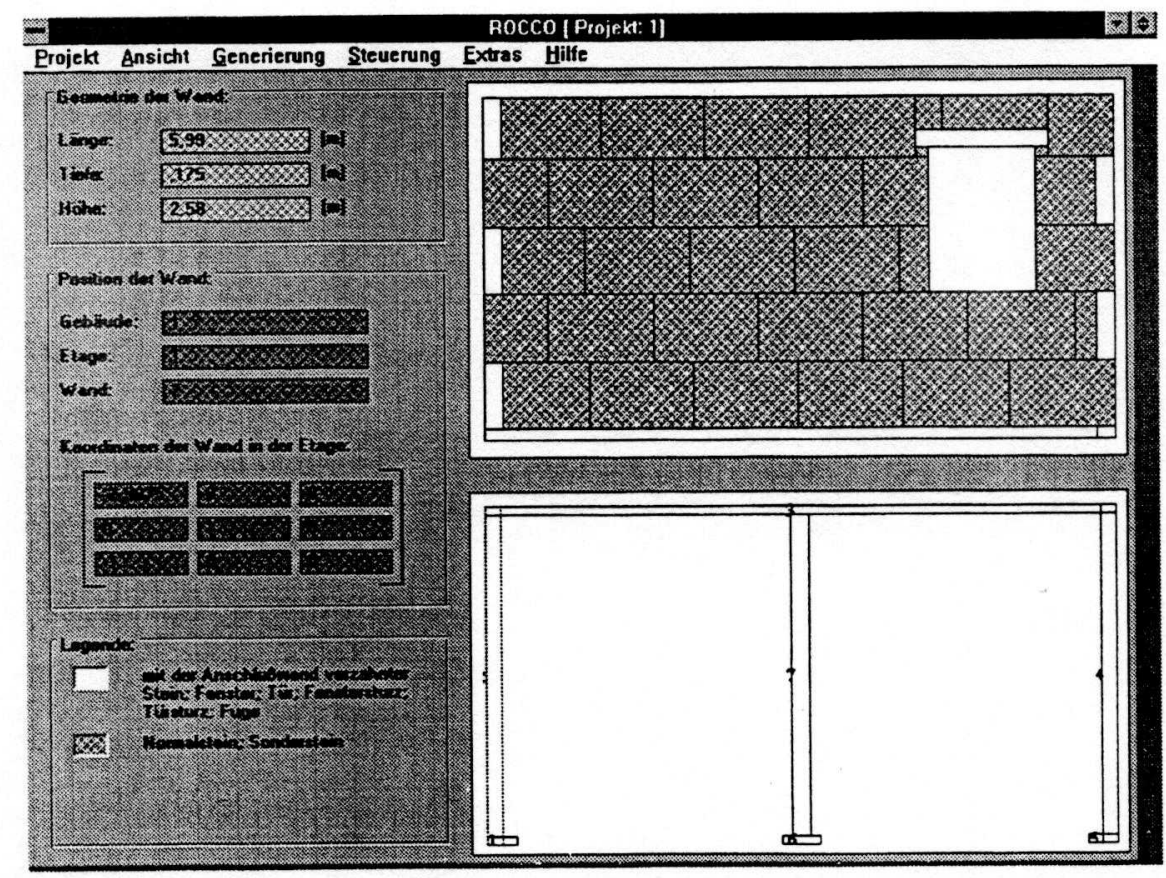

Fig. 5: user interface of the masonry programming system 
In order to determine the occuring cases for positioning operations algorithms have to be implemented which direction of assembly is necessary. Therefore, various transformations of coordinates regarding the robot system have to be made at first. The reason is that all elements, geometric data and positions are represented in the respective coordinate system. Finally, it has to be considered that a whole building or floor has to be completed by taking a number of working points.

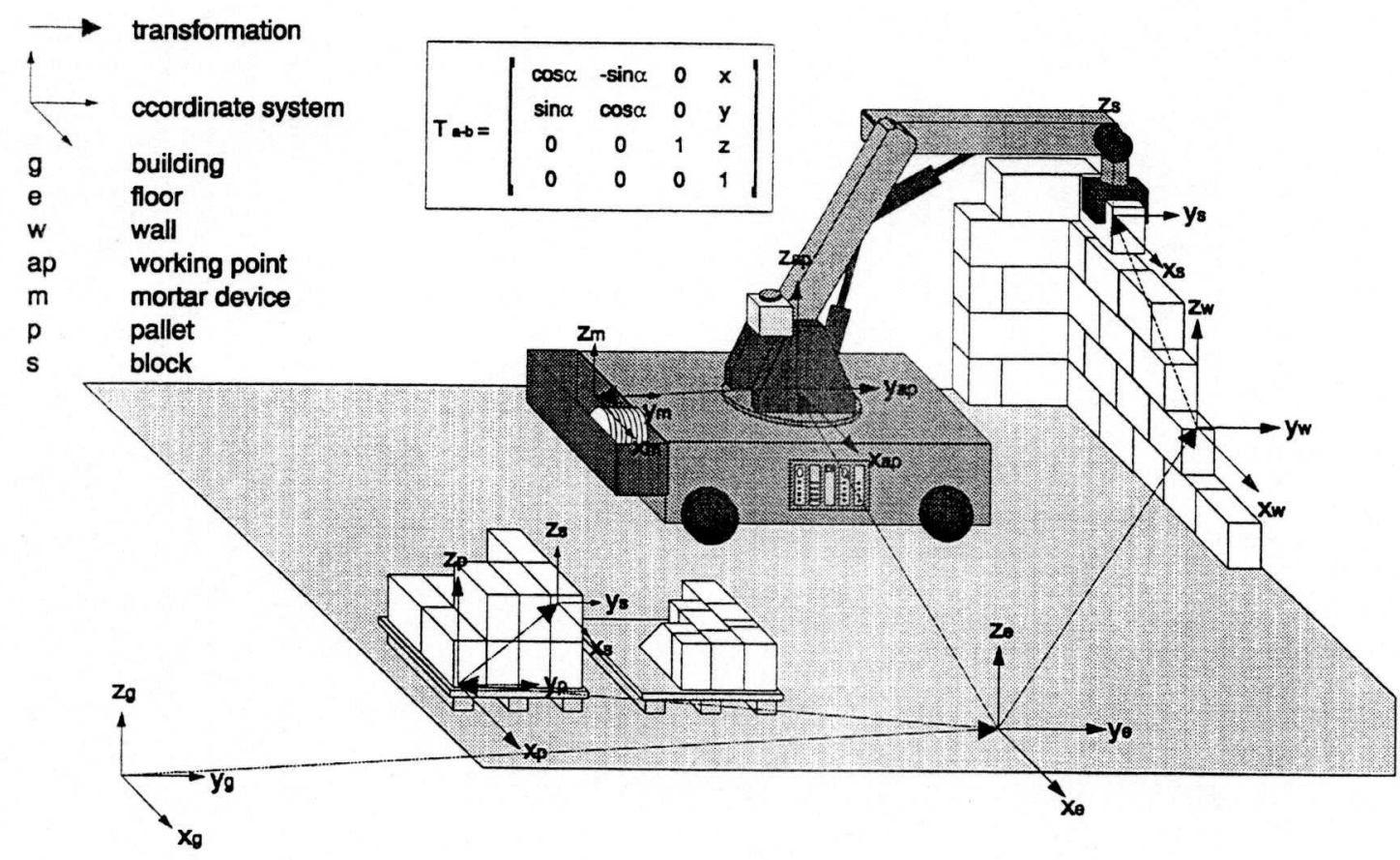

Fig. 6: relationships between the necessary coordinate systems

In order to determine the assembly strategy and direction for the following path planning existing and possible neighbouring elements of the wall have to be recognized. The essential point is the formation of a lot of boundary elements of each existing wall group. So, the focus lays on a specification of current borders of the respective wall groups because it is possible that several walls in compound structure are built in one working point of the robot. As a result of comparing this lot with the block to be assembled in vertical direction the basic examination for the path planning is done. Therefore, a specification of neighbouring wall elements by their corner points as well as minimum and maximum z-dimension in floor coordinates is necessary at first. Secondly, the contemplation is limited through a enveloping cylinder of the block to be assembled. The next steps are the determination of the vertical 
neighbourhood by a comparison of the vertical minimum and maximum levels, the determination of the number and location of neighbouring blocks to detect the correct assembly strategy and at last the updating of the boundary lot.

In order to detect the respective assembly direction the working point of the robot and the block to be assembled must be analysed with regard to the wall coordinate system. So, to set a regular block in a straight wall four possible cases occur on the assumption that the wall coordinate system may take up two orientations. These cases are used for both the calculation of the coarse positions and the calculation of the relative movements for exact positioning. Further examinations have to be done to assemble irregular blocks as well as corner blocks. To handle irregular i.e. cutted blocks the assembly direction already influences the gripping at the pallet because the block cannot be handled in the middle but according to the assembly direction towards ist next neighbouring block. To detect the correct case of corner positions there are two different requirements. The actual block and the block before must be elements of two different walls and addtionally the actual block must be signed as a so called other element in the second one. Another constraint is that the actual block and the following one must be elements of different walls. These two requirements lead also to two different postioning strategies (see figure 7).

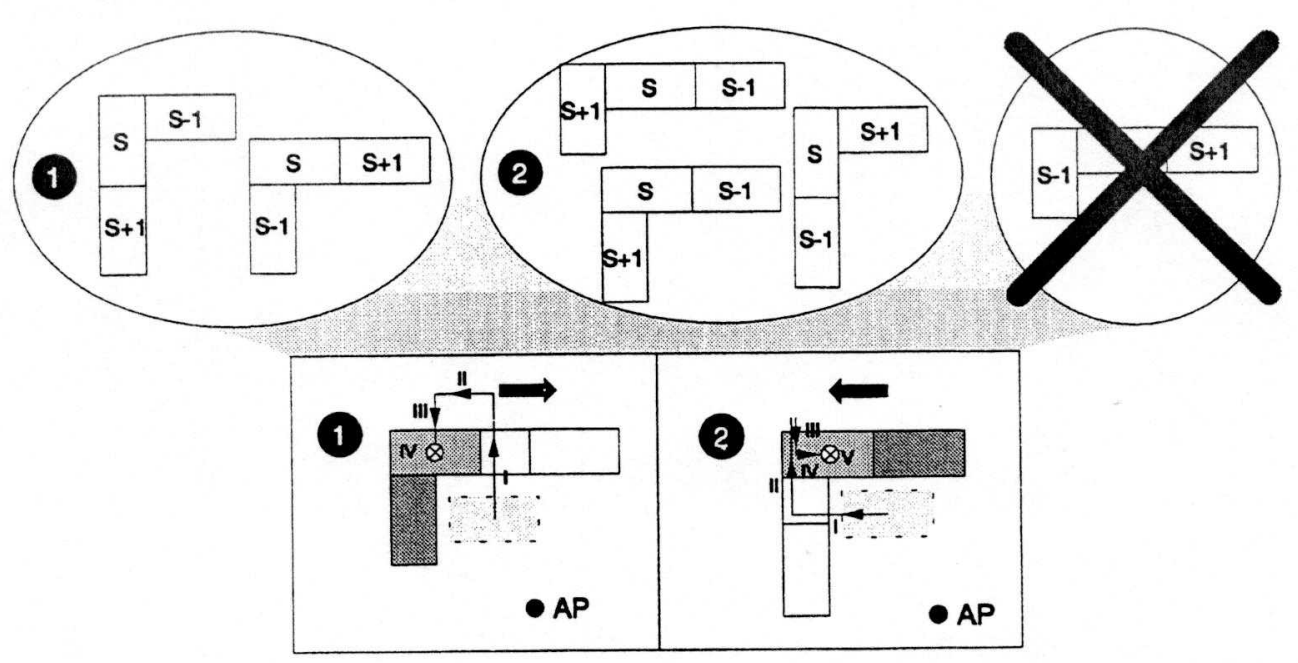

Fig. 7: basic path planning by the example of corner blocks 


\subsection{Programming System for Wall Slits}

To realise an efficient programming of the conceptuated indoor robot for residential finishing tasks like wall processing in particular (i.e. installations, roughcasting, tiling) other dominating preconditions have to be considered. In this case of robot application such small construction companies for tasks beyond masonry can hardly be integrated in a continuous CAD/RC-process. Thus for a high acceptance by the user it is necessary to create programming systems with a higher flexibility for the onsite employment according to the workers' qualifications. So, a more detailed work preparation and the programming itself should be done on the construction site. Therefore, different possibilities are carried out roughly devided into sensor guided systems and graphic oriented programming systems, that leads either to a great effort of preparing markings at the wall or an assessable effort of input. Since the use of sensor guided systems restricts intended alternative applications a graphical, interactive programming system becomes appropriate. To cut slits for electrical installations by the employment of the indoor robot, the first manual inputs are the respective wall dimensions and the positions of doors and windows. The next step is to enter the wiring on a PC by drawing the respective courses (see figure 8). Assuming a higher integrated construction process in the future it will be possible to use the wall data of the masonry application in order to reduce the effort of input.

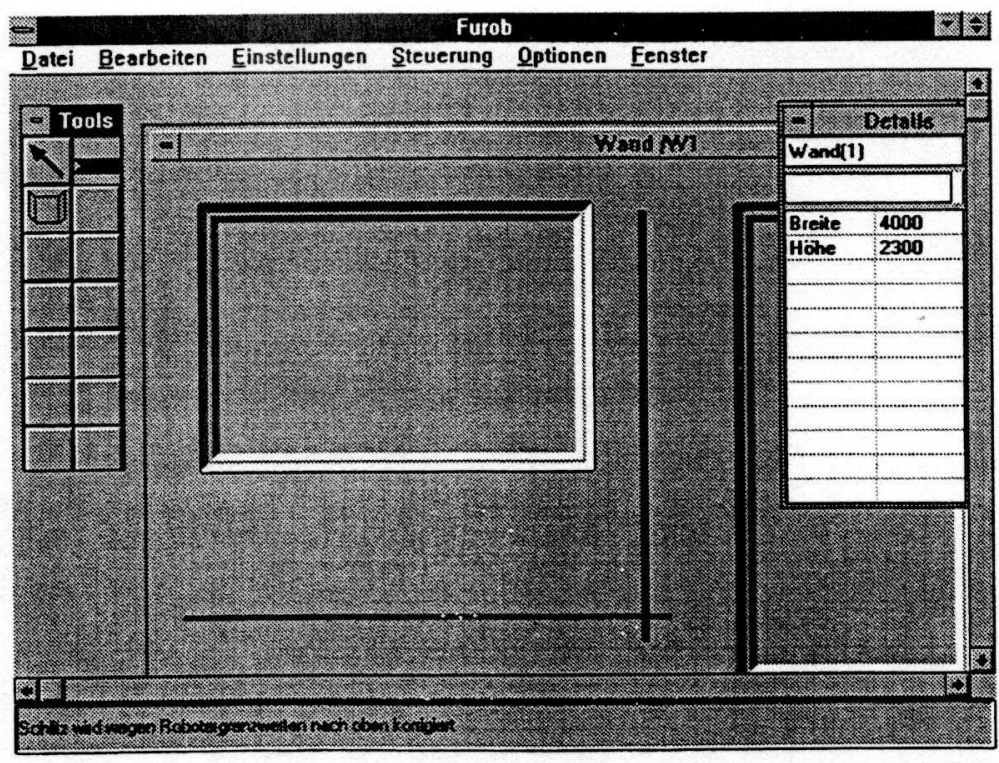

Fig. 8: graphical user interface for the installation application 
In the opposite to the masonry programming system the path planning needs no module to determine basic manufacturing strategies because the principle defaults whether corner manufacturing is necessary or not is given by the construction worker. However, a detailed specification of technological data for the orientations of the hand axes is done by the system according to the limitations through neighbouring walls. So, to calculate all necessary coordinates for the path planning distinctions are made between horizontal and vertical slits and whether they are situated near to a right or left corner or near to the floor or the ceiling. The manufacturing process done by a joint cutter is designed to minimize manual refinishings so that in general, ordinary horizontal and vertical slits are V-shaped. So, the specific procedure shown exemplarily in figure 8 has to be parameterized.

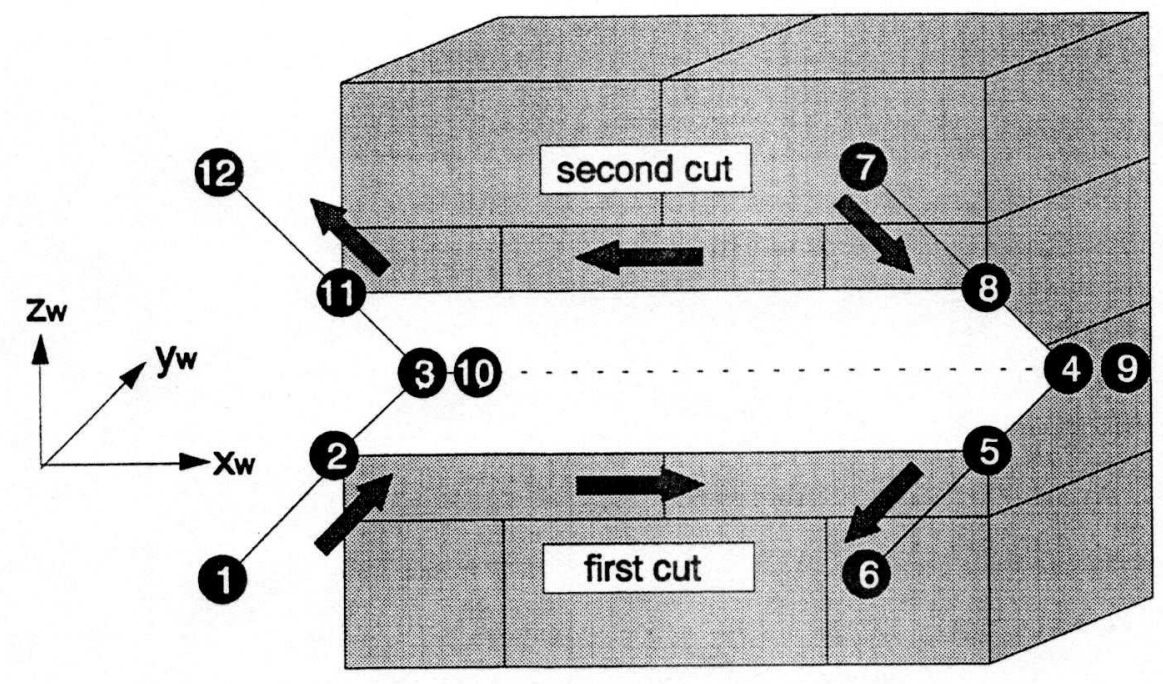

Fig. 9: basic operating sequence for horizontal slits

Furthermore, four different cases of the corner manufacturing itself have to be considered. All these specifications are unalterable on account of the strong limitations by the collision volumes within closed rooms. Here, the basic manufacturing strategy consists of two horizontal cuttings.

After the determination of the kind of slit a verfication is performed if the specifications are within the required installation zones and within the robots limitations. Moreover, prohibited collisions with doors, windows and other slits are checked. Eventually, the specifications of the user are adjusted automatically. To ascertain the detailed path planning the kind of cable and the number of cables within one slit has to be selected. Hence, the insertion depth 
depending on the distance to the wall can be calculated. In particular, to process corners the minimum inside radius of cables has to be taken into account as well.

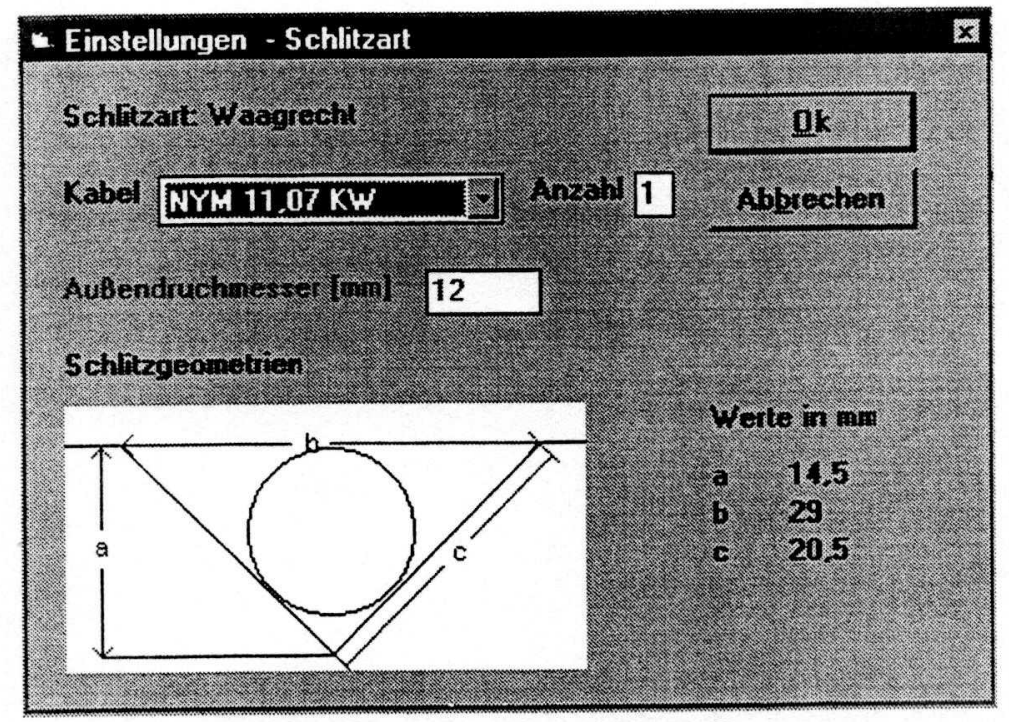

Fig. 10: selection of cables and diameters

To start from left, lower bound the operating sequence regarding different working positions is specified automatically. For the execution of the robot programmes functional macros are used as well. They will be also parametrized depending on the input data of the construction worker or electrician and the calculations by the generation module, so that the method of processing is rather similar to the masonry application.

\section{Conclusions}

The article expounds the integration of automated construction processes and the necessary work preparation tasks regarding the development of two robot applications to meet rationalisation and flexibilty in the building industry in the forseeable future. The essential parts are the conceptuation and realisation of the respective programming systems and the organization of the communication processes on the construction site.

In the future the shown system should be open for higher integration of construction tasks. So, it can be possible to use the generated data of the masonry construction for indoor applications as well. But for the moment, single and efficient solutions should be feasible despite the existing preconditions in the building industry. The next step is the intention to realise the interior 
robot together with industrial partners within a national project funded by the German Federal Ministry of Education, Research and Technology.

\section{References}

[1] Soeffner, F.: Baukonjunktur in Westeuropa. ifo-Schnelldienst 21/95. Institut fuer Wirtschaftsforschung: Muenchen, 1995, pp. 15-19

[2] Statistisches Bundesamt: Ausgewaehlte Zahlen fuer die Bauwirtschaft 1994. MetzlerPoeschel: Stuttgart, 1995

[3] Weule, H.; Spath, D.; Andres, J.: Flexible Automation in Masonry Construction. Production Engineering Vol. II/2. Hanser: Muenchen, Wien, 1995, pp. 163-166

[4] Spath, D.; Andres, J.; Bock, T.; Steffani, H.F.: Flexible Automatisierung im Mauerwerksbau. Autonome Mobile Systeme 1994. Spinger: Berlin, Heidelberg, 1994, pp. 306-315

[5] Spath, D.; Andres, J.: ROCCO, ein Serviceroboter fuer die Baubranche. TR Transfer No. 13,1996 , pp. $18-22$

[6] Spath, D.; Andres, J.: Concept of a Robot for Interior Building Trades by the Example of Wall Slits in Masonry. Proceedings of the 13th ISARC, Tokyo, 11-13 June 1996, pp. $941-948$

[7] Abdul Sater, H.; Delchambre, A.: Computer-Aided Planning in Masonry Construction. Proceedings of the 11th ISARC, Brighton, 24-26 May 1994. Elsevier: Amsterdam, 1994, pp. 103-110 